\title{
Comparison of long non-coding RNA expression profiles in human dental follicle cells and human periodontal ligament cells
}

\author{
LIPING WU $^{1 *}$, LIDI DENG $^{1 *}$, HONG HONG $^{2}$, CAIXIA PENG ${ }^{1}$, \\ XUEQIN ZHANG ${ }^{1}$, ZHENGYUAN CHEN $^{1}$ and JUNQI LING ${ }^{3}$ \\ ${ }^{1}$ Department of Orthodontics; ${ }^{2}$ Zhujiang New Town Dental Clinic; ${ }^{3}$ Department of Endodontics, \\ Guanghua School of Stomatology, Guangdong Provincial Key Laboratory of Stomatology, \\ Sun Yat-Sen University, Guangzhou, Guangdong 510055, P.R. China
}

Received July 9, 2018; Accepted February 28, 2019

DOI: $10.3892 / \mathrm{mmr} .2019 .10308$

\begin{abstract}
The dental follicle develops into the periodontal ligament, cementum and alveolar bone. Human dental follicle cells (hDFCs) are the precursor cells of periodontal development. Long non-coding RNAs (lncRNAs) have been revealed to be crucial factors that regulate a variety of biological processes; however, whether lncRNAs serve a role in human periodontal development remains unknown. Therefore, the present study used microarrays to detect the differentially expressed lncRNAs and mRNAs between hDFCs and human periodontal ligament cells (hPDLCs). A total of 845 lncRNAs and 1,012 mRNAs were identified to be differentially expressed in hDFCs and hPDLCs (fold change $>2.0$ or $<-2.0 ; \mathrm{P}<0.05)$. Microarray data were validated by reverse transcription-quantitative polymerase chain reaction. Bioinformatics analyses, including gene ontology, pathway analysis and coding-non-coding gene co-expression network analysis, were performed to determine the functions of the differentially expressed lncRNAs and mRNAs. Bioinformatics analysis identified that a number of pathways may be associated with periodontal development, including the p53 and calcium signaling pathways. This analysis also revealed a number of lncRNAs, including NR_033932, T152410, ENST00000512129, ENST00000540293, uc021sxs.1 and ENST00000609146, which may serve important roles in the biological process of hDFCs. In addition, the lncRNA termed maternally expressed 3 (MEG3) was identified to be differentially expressed in hDFCs by
\end{abstract}

Correspondence to: Professor Junqi Ling, Department of Endodontics, Guanghua School of Stomatology, Guangdong Provincial Key Laboratory of Stomatology, Sun Yat-Sen University, 56 Lingyuanxi Road, Guangzhou, Guangdong 510055, P.R. China E-mail: lingjq@mail.sysu.edu.cn

${ }^{*}$ Contributed equally

Key words: long non-coding RNA, dental follicle, periodontal ligament, microarray analysis reverse transcription-quantitative polymerase chain reaction. The knockdown of MEG3 was associated with a reduction of pluripotency makers in hDFCs. In conclusion, for the first time, to the best of our knowledge, the current study determined the different expression profiles of IncRNAs and mRNAs between hDFCs and hPDLCs. The observations made may provide a solid foundation for further research into the molecular mechanisms of lncRNAs in human periodontal development.

\section{Introduction}

The dental follicle, originating from the cranial neural crest, is a loose ectomesenchyme-derived tissue, which surrounds the dental papilla and enamel organ during the development of teeth. The dental follicle develops into periodontal supporting tissues, which serve important functions, including in support, buffering, rebuilding and regeneration (1). hDFCs exhibit a high capacity for proliferation, self-renewal and multi-directional differentiation, and form structures, including the cementum and periodontal ligament (PDL), when subcutaneously transplanted into immunodeficient mice $(2,3)$. hDFCs share a similar phenotype with human PDLCs (hPDLCs), including a mineralization ability, and they also exhibit apparent embryonic characteristics, including pluripotency, heterogeneity and a higher potential for cementum formation in vivo (4-6). It has been suggested that hDFCs may provide a new source of seed cells for stem cell therapy and periodontal tissue engineering. Therefore, understanding the key target genes and underlying molecular mechanisms of hDFC differentiation is required for promoting periodontal development and regeneration.

Long non-coding RNAs (lncRNAs) are defined as non-protein coding RNA molecules that are $>200$ nucleotides long (7). IncRNAs perform their biological effects through a number of mechanisms, including genetic imprinting, chromatin remodeling, cell cycle regulation, splicing and mRNA inactivation. IncRNAs control the pluripotency and stemness of embryonic stem cells and induced pluripotent stem cells, or promote the differentiation of pluripotent cells in the opposite manner. Additionally, lncRNAs may transcriptionally or post-transcriptionally regulate gene expression via different molecular mechanisms $(8,9)$. 
An increasing number of studies indicate that lncRNAs serve critical roles in the development of organs, including the brain (10), heart (11), liver (12), lungs (13) and bone (14). lncRNAs also serve significant roles in tooth development. For example, the lncRNA differentiation antagonizing non-protein coding RNA (DANCR) serves a role in reparative dentin formation and regenerative endodontics (15). DANCR is an essential mediator in the proliferation and differentiation of dental tissue-derived stem cells, including dental pulp stem cells, stem cells from the apical papilla and periodontal ligament stem cells (PDLSCs) (16). hDFCs and hPDLCs are essential cells in different stages of periodontal development. However, it remains unclear what potential roles IncRNAs serve in periodontal development and whether lncRNAs are involved in specific activities in different cells. Therefore the current study used microarrays to obtain the different expression profiles of lncRNAs and mRNAs between hDFCs and hPDLCs. Furthermore, the microarray data were validated by reverse transcription-quantitative polymerase chain reaction (RT-qPCR). Bioinformatics analyses were performed to predict the possible roles of the differentially expressed IncRNAs and mRNAs in periodontal development. The results demonstrated that lncRNAs may serve critical roles in periodontal development, and provided a solid foundation for further research.

\section{Materials and methods}

Cell culture. Human dental follicle and periodontal ligament samples were obtained from four adolescents ( 2 males and 2 females) between 12 and 16 years old following premolar and immature impacted third molar (roots developed to $<2 / 3$ their full size) extraction for orthodontic reasons. No significant differences were identified in age or sex. Participants included in the study had no history of systemic disease, smoking or specific medication. Tooth extraction was performed at the Hospital of Stomatology, Sun Yat-Sen University (Guangzhou, China) between June 2017 and July 2017. All experimental protocols were conducted under the guidelines set by the Sun Yat-Sen University Ethics Committee and written informed consent was obtained from all patients and their parents. hDFCs and hPDLCs were isolated as previously described $(17,18)$. Briefly, dental follicle tissues were gently removed with a scalpel from where they attached to the root dentin and were digested in a solution containing $1 \mathrm{U} / \mathrm{ml}$ collagenase type I (Sigma-Aldrich; Merck KGaA, Darmstadt, Germany), hyaluronidase (Sigma-Aldrich; Merck KGaA, Darmstadt, Germany) and 1 U/ml DNase I (Roche Applied Science, Mannheim, Germany). Periodontal ligament tissues were isolated from the middle one-third of the root surface and digested in a solution of $3 \mathrm{mg} / \mathrm{ml}$ collagenase type I (Sigma-Aldrich; Merck KGaA) and $4 \mathrm{mg} / \mathrm{ml}$ dispase (Sigma-Aldrich; Merck KGaA). hDFCs and hPDLCs were cultured in Dulbecco's modified Eagle's medium supplemented with $20 \%$ fetal bovine serum (both Gibco; Thermo Fisher Scientific, Inc., Waltham, MA, USA), $100 \mathrm{U} / \mathrm{ml}$ penicillin and $100 \mu \mathrm{g} / \mathrm{ml}$ streptomycin (Sigma-Aldrich; Merck KGaA) at $37^{\circ} \mathrm{C}$ in $5 \% \mathrm{CO}_{2}$. Every $2-3$ days, the medium was replaced. When cells reached $80 \%$ confluence, they were passaged at a 1:3 ratio using $0.25 \%$ trypsin/EDTA (Gibco; Thermo Fisher
Scientific, Inc.). Third-generation cells were used for the subsequent experiments.

RNA extraction. Total RNA was extracted using TRIzol ${ }^{\circledR}$ reagent (Invitrogen; Thermo Fisher Scientific, Inc.). Quantification and quality checks were conducted using a NanoDrop ND-1000 spectrophotometer (Thermo Fisher Scientific, Inc.). RNA integrity and genomic DNA contamination were determined by denaturing agarose gel electrophoresis.

RNA microarray. The total RNA was purified using an RNeasy Mini kit (Qiagen GmbH, Hilden, Germany) and labeled with a Quick Amp Labeling kit (Agilent Technologies, Inc., Santa Clara, CA, USA). Labeled RNA was purified again using the RNeasy Mini kit. The RNA was subsequently hybridized onto an Arraystar Human lncRNA Expression Microarray (version 4.0; Arraystar, Inc., Rockville, MA, USA), which was designed for 30,586 lncRNAs and 26,109 coding genes based on the RefSeq (https://ncbi.nlm.nih.gov/refseq/), UCSC Known Genes and Gencode (https:/genome.ucsc. edu/) and Ensembl databases (http://ensemblgenomes.org/). Agilent Feature Extraction software (version 11.0.1.1; Agilent Technologies, Santa Clara, CA, US) was used to analyze the acquired array images. The microarray data were deposited in the Gene Expression Omnibus (GEO; https://www.ncbi. nlm.nih.gov/geo/) and are accessible through GEO Series accession no. GSE124352. Differentially expressed lncRNAs and mRNAs were identified to be statistically significant (fold change $>2.0$ or $<-2.0 ; \mathrm{P}<0.05$ ) using a paired t-test. The microarray was performed by Kangchen BioTech Co., Ltd. (Shanghai, China). Subgroup analysis was conducted to classify the differentially expressed lncRNAs according to their expression levels.

RT-qPCR analysis. Total RNA of hDFCs and hPDLCs was reverse transcribed into complementary DNA using an RT kit (Takara Bio, Inc., Otsu, Japan), conducted at $37^{\circ} \mathrm{C}$ for $15 \mathrm{~min}$ and $85^{\circ} \mathrm{C}$ for $5 \mathrm{sec}$. In total, six lncRNAs and six mRNAs were randomly selected for RT-qPCR analysis using the RANDBETWEEN function in Microsoft Excel (version 2010, Microsoft Corporation, Redmond, WA, USA). RT-qPCR was performed using SYBR Green Real-Time PCR Master mix (Invitrogen; Thermo Fisher Scientific, Inc.), and was run at $95^{\circ} \mathrm{C}$ for $5 \mathrm{~min}$, followed by 40 cycles at $95^{\circ} \mathrm{C}$ for $10 \mathrm{sec}, 65^{\circ} \mathrm{C}$ for $20 \mathrm{sec}$ and $72^{\circ} \mathrm{C}$ for $30 \mathrm{sec}$. Primers were synthesized by Invitrogen; Thermo Fisher Scientific, Inc. (Table I). The characteristics of the lncRNAs are presented in Table II. Data were normalized to GAPDH and the relative level of gene expression was calculated using the $2^{-\Delta \Delta \mathrm{Cq}}$ method (19).

Bioinformatics analyses. Gene ontology (GO; http://geneontology.org/) was conducted to analyze the functions of the differentially expressed genes. GO analysis examines gene regulatory networks based on biological processes, cellular components and molecular functions (20). A $\chi^{2}$ test and a two-sided Fisher's exact test were used to classify the GO category. Enrichment of the GO category was determined by the significance of the functions. Kyoto Encyclopedia 
Table I. Primers used for the reverse transcription-quantitative polymerase chain reaction.

\begin{tabular}{lll}
\hline Gene name & \multicolumn{1}{c}{ Forward (5'-3') } & Reverse $\left(5^{\prime}-3^{\prime}\right)$ \\
\hline NR_033917 & TCACTGTCATGCACTAGCGG & CAGCCCTTGAGGTTGTCCTT \\
NR_038367 & CCCACCGTTCAATGAAAG & GTTTCAAACACCCACATTTC \\
NR_026861 & ACTCGCCTTTTGCGAGAAGA & TCGCAGTTTCCTAGTGGGAC \\
NR_102703 & AGCTGTGAACGGTAGCAGTG & AGGCAGTTTGTGGGTCAGT \\
NR_110162 & GGCCCATTCCTGTGAATCGT & GTGACTCAAAGCAGCAGAAGC \\
ENST00000430859 & CCCCTTAGCCTGCTTGTGAT & CCAGTGAAAACTGCACAGAGC \\
KCNK12 & CATGTACACCAGCGTGGAGG & AGCGAGTAAATGCAGCACAC \\
CCL11 & TCCCTGGAATCTCCCACACT & CACTCAGGCTCTGGTTTGGT \\
MDK & TCAGACCGGTTCTGGAGACA & TTTGCTTTGGTCTTGGGGGT \\
SAA2 & GAGTGGCAGAGACCCCAATC & TCAGCTTCTCTGGACATAGACC \\
MGST1 & CGGCCTCACCATTCCAGAC & CAAGGTCATTCAGGTGGGCT \\
HIST1H2BG & GCAGTGTCCGAAGGTACCAA & TGTGAGACTTGAGTGGCTCTG \\
GAPDH & CTGGGCTACACTGAGCACC & AAGTGGTCGTTGAGGGCAATG
\end{tabular}

KCNK12, potassium two pore domain channel subfamily $\mathrm{K}$ member 12; CCL11, C-C motif chemokine ligand 11; MDK, midkine; SAA2, serum amyloid A2; MGST1, microsomal glutathione S-transferase 1; HIST1H2BG, histone cluster 1 H2B family member g.

Table II. Characteristics of lncRNAs for validation.

\begin{tabular}{|c|c|c|c|c|c|c|}
\hline lncRNA & Expression & Chromosome & Strand & Start & End & Class \\
\hline NR_033917 & Down & Chr20 & + & 4173736 & 4176600 & Intergenic \\
\hline NR_038367 & Down & Chr7 & + & 27135712 & 27139877 & Antisense \\
\hline NR_026861 & Down & Chr6 & - & 166337535 & 166401527 & Intergenic \\
\hline NR_102703 & Up & Chrx & - & 149007562 & 149025779 & Undefined \\
\hline NR_110162 & Up & Chr7 & - & 112594689 & 112635698 & Undefined \\
\hline ENST00000430859 & Up & Chr7 & + & 20257208 & 20261315 & Bidirectiona \\
\hline
\end{tabular}

lncRNA, long non-coding RNA; Chr, chromosome.

of Genes and Genomes (https://www.genome.jp/kegg/) pathway analysis of the differentially expressed mRNAs was performed to identify the associated pathways. Connections between the genes were revealed based on their associations in the identified pathways. In addition, a coding-non-coding gene co-expression (CNC) network was constructed to investigate the interactions between the differentially expressed lncRNAs and mRNAs. A number of differentially expressed mRNAs were identified to be associated with development. Pearson's correlation coefficient was calculated for each pair of lncRNAs and mRNAs, and the most significantly correlated genes (Pearson's correlation coefficient $\geq 0.90$ or $\leq-0.90$; $\mathrm{P}<0.05)$ were selected to construct the network. The network was generated using Cytoscape software (v2.8.2; https://cytoscape.org/). The default annotation categories were selected in the software and an enrichment score $>2.0$ was considered significant.

Transient transfection. hDFCs were transfected with $50 \mathrm{nM}$ MEG3-small interfering (si)RNA and NC-siRNA using Lipofectamine ${ }^{\circledR} 3000$ (Invitrogen; Thermo Fisher Scientific, Inc.) at a density of $1.5 \times 10^{5}$ cells, according to the manufacturer's protocol. The MEG3-siRNA and negative control (NC)-siRNA were synthesized by Guangzhou RiboBio Co., Ltd. (Guangzhou, China). The MEG3-siRNA sequences were as follows: GACTTAAACCAATGCCCTA, CCTCTT ACCTAAAGACTTA, CCCTCTTGCTTGTCTTACT. The expression of pluripotency-associated genes was detected by RT-qPCR $72 \mathrm{~h}$ post-transfection.

Statistical analysis. Data are presented as the mean \pm standard deviation $(n=3)$ and were analyzed using a Student's t-test. All statistical analysis was performed with SPSS 20.0 (IBM Corp., Armonk, NY, USA). A fold-change $>2.0$ or $<-2.0$ and $\mathrm{P}<0.05$ was considered to indicate a statistically significant difference.

\section{Results}

lncRNA and mRNA expression profiles of $h D F C s$ and $h P D L C s$. Gene expression patterns of hDFCs and hPDLCs were screened with the high-throughput microarray method to reveal potential molecular mechanisms underlying hDFC development into periodontal tissues. Microarray probes detected 36,430 transcripts in hDFCs and hPDLCs (Fig. 1). 

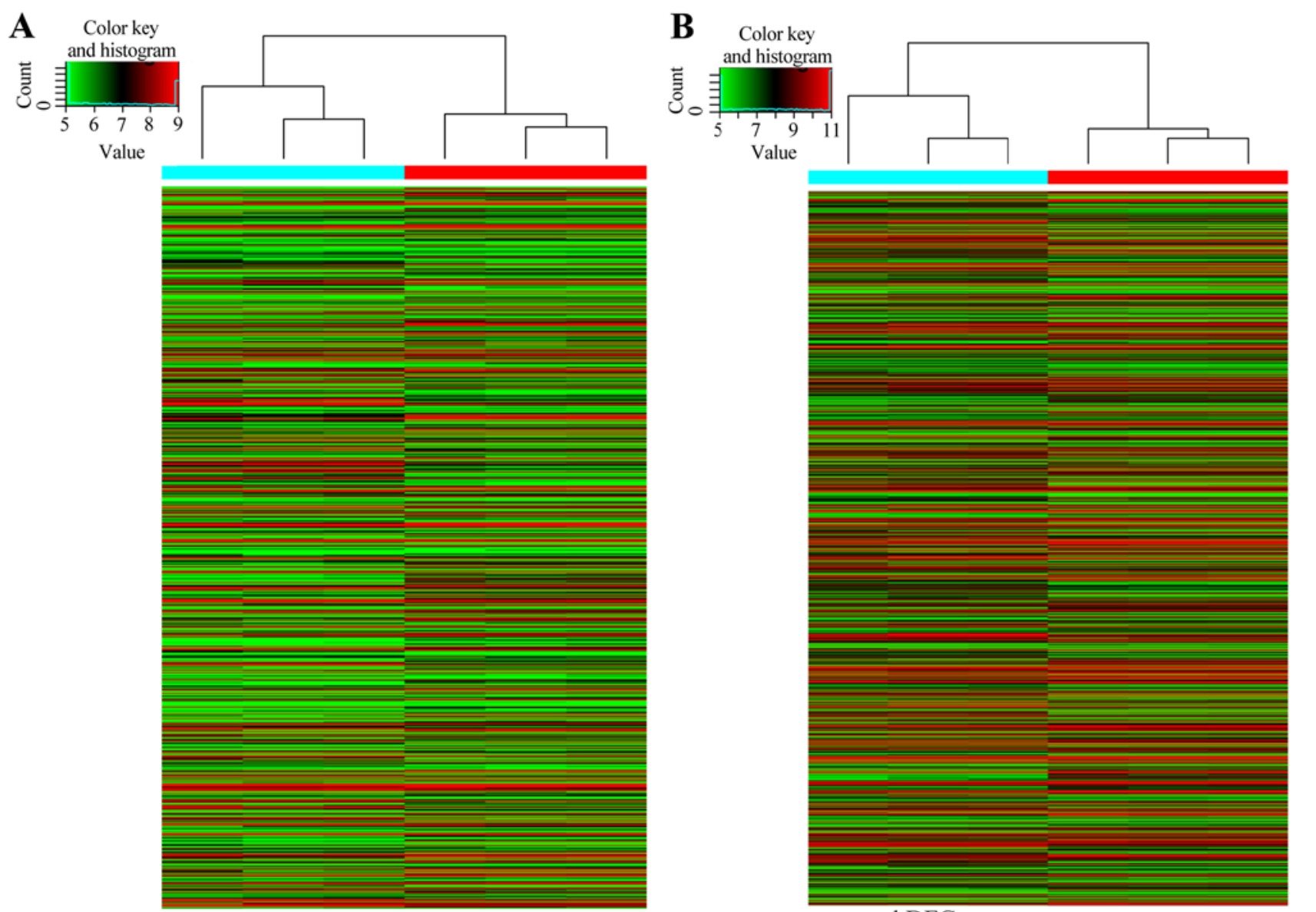

hDFCs

hPDLCs

hDFCs

hPDLCs

Figure 1. Expression profiles of lncRNAs and mRNAs in hDFCs and hPDLCs. (A) Hierarchical clustered heat maps of differentially expressed lncRNAs. (B) Hierarchical clustered heat maps of differentially expressed mRNAs. Each column represents one sample and 'Value' indicates the gene expression. The intensity of the color indicates the expression level, with red representing a high expression level and blue representing a low expression level. 'Count' refers to the number of genes that are expressed at the same level. lncRNA, long non-coding RNA; hDFC, human dental follicle cell; hPDLC, human periodontal ligament cell.

A total of 845 lncRNAs were identified to be differentially expressed in the hDFCs and hPDLCs, of which 460 were upregulated and 385 were downregulated in the hDFCs compared with the hPDLCs. In addition, a total of 1,012 mRNAs (6.12\%) were differentially expressed, 553 mRNAs were upregulated and 459 mRNAs were downregulated in the hDFCs compared with the hPDLCs. A scatter plot was generated to visualize the variation in expression of lncRNAs and mRNAs in the hDFCs and hPDLCs (Fig. 2A and B). Additionally, a volcano plot was used to visualize significantly differentially expressed lncRNAs and mRNAs (Fig. 2C and D). Furthermore, the microarray results revealed the top ten differentially expressed lncRNAs and mRNAs (Tables III and IV).

Subgroup analysis classified the differentially expressed lncRNAs as intergenic lncRNAs (lincRNAs) and antisense lncRNAs (ASlncRNAs). The nearby protein-coding genes were identified to predict potential functions of lncRNAs. A total of 113 lincRNAs and 35 ASlncRNAs, including NR_033932, T152410, ENST00000512129 and ENST00000540293, were revealed to be located near known protein-coding genes.

To verify the reliability of the microarray data, six differentially expressed lncRNAs and six differentially expressed mRNAs were randomly selected for analysis of their expression levels by RT-qPCR. Each differentially expressed gene was numbered and the RANDBETWEEN function in Microsoft Excel was used. Compared with hPDLCs, hDFCs exhibited decreased expression levels of the lncRNAs NR_033917, NR_038367 and NR_026861, and the mRNAs potassium two pore domain channel subfamily $\mathrm{K}$ member 12 , C-C motif chemokine ligand 11 and midkine. By contrast, hDFCs demonstrated increased expression levels of the lncRNAs NR_102703, NR_110162 and ENST00000430859, and the mRNAs serum amyloid A2, microsomal glutathione S-transferase 1 and histone cluster 1 H2B family member g (Fig. 3). These results were consistent with the microarray data.

GO analysis and pathway analysis. GO analysis revealed functions associated with the differentially expressed genes and provided annotations to describe the genes and gene products (Fig. 4). The top five downregulated GO functions were the following: 'Complex of collagen trimers'; 'endothelial cell morphogenesis'; 'penile erection'; 'positive regulation of renal sodium excretion'; and 'positive regulation of stem cell differentiation' (Fig. 5A). The top five upregulated GO functions were as follows: 'Condensed chromosome outer kinetochore'; 'aldehyde dehydrogenase activity'; 'regulation of mononuclear 

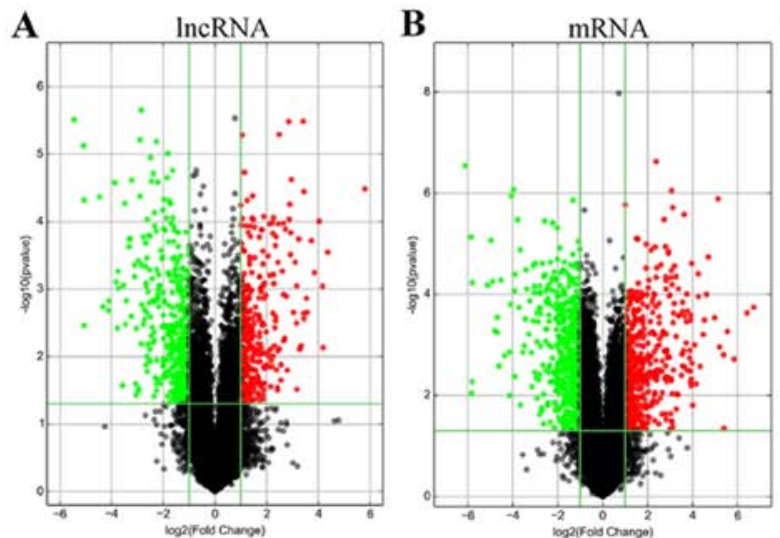

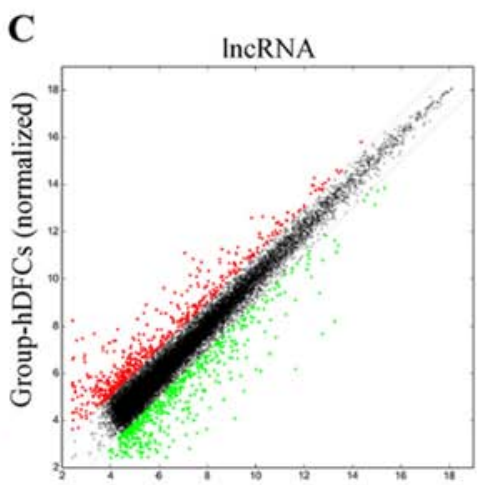

Group-hPDLCs (normalized)

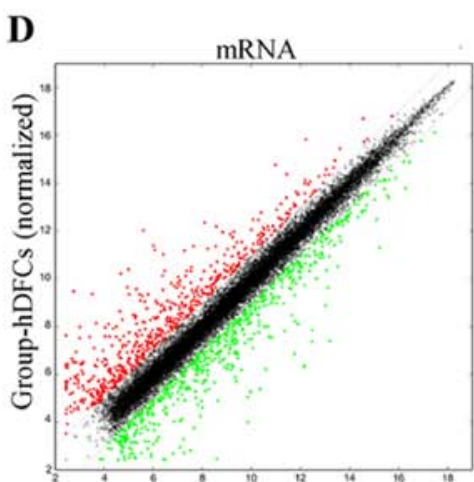

Group-hPDLCs (normalized)

Figure 2. Scatter plots and volcano plots of differentially expressed genes. Scatter plots of significantly differentially expressed (A) lncRNAs and (B) mRNAs The $\mathrm{x}$-axis represents the mean normalized $\log 2$ fold-change for the hPDLCs and the y-axis represents the mean normalized log 2 fold-change for the hDFCs. Volcano plots were generated to visualize the differentially expressed (C) lncRNAs and (D) mRNAs. The x-axis indicates the log2 fold-change and the $\mathrm{y}$-axis represents the $\log 2 \mathrm{P}$-values. The green and red points indicate the upregulated and downregulated lncRNAs and mRNAs with statistical significance, respectively. lncRNA, long non-coding RNA; hDFC, human dental follicle cell; hPDLC, human periodontal ligament cell.

cell migration'; 'mononuclear cell migration'; and 'condensed nuclear chromosome and centromeric region' (Fig. 5B).

Pathway analysis revealed 20 downregulated pathways. The top three enriched networks were 'rheumatoid arthritis', 'ECM-receptor interaction' and 'arrhythmogenic right ventricular cardiomyopathy (ARVC)' (Fig. 6A). In addition, 36 upregulated pathways were identified and the top three enriched networks were 'p53 signaling pathway', 'oocyte meiosis' and 'renin secretion' (Fig. 6B).

CNC network. A total of 615 lncRNAs and 16 mRNAs were selected to construct the CNC network (Fig. 7). The CNC network included 488 positive pairs and 438 negative pairs, and each mRNA could be associated with 1-20 lncRNAs, and vice versa. The CNC network indicated the different molecular mechanisms of hDFCs compared with hPDLCs associated with the inter-regulation of lncRNAs and mRNAs.

MEG3 regulates the pluripotency of hDFCs. MEG3 was reported to serve a crucial role in initiating embryogenesis and development (21). The current study depleted MEG3 expression in hDFCs and identified a reduction in the transcription of pluripotency-associated genes (Fig. 8). In summary, these results may suggest a possible regulatory role of MEG3 in the pluripotency of hDFCs.

\section{Discussion}

The dental follicle contains precursor cells that may develop into periodontal ligament, cementum and alveolar bone during periodontal development. hDFCs and hPDLCs are the major cells of the dental follicle and periodontal ligaments, respectively. These cells are key markers that represent different stages of periodontal tissue development; however, hDFCs exhibit more obvious embryonic characteristics, including pluripotency and heterogeneity. Therefore, understanding the mechanisms associated with the differentiation of hDFCs to hPDLCs is crucial for promoting periodontal development and regeneration.
An increasing number of studies have indicated that numerous lncRNAs regulate cell differentiation through epigenetics or in-cis/in-trans gene transcription. Previous studies have demonstrated that differential expression levels of lncRNAs are associated with human diseases and biological processes. IncRNAs serve vital roles in cell development and lineage commitment. Therefore, lncRNAs may serve as key regulators of human tissue development and regeneration (22). lncRNAs are differentially expressed during the differentiation of human bone marrow mesenchymal stem cells and PDLSCs $(23,24)$. To investigate the changes and roles of lncRNAs during the development of hDFCs, the current study used high-throughput microarrays to detect the expression levels of genes. Only the middle one-third of the root surface was taken while obtaining the periodontal ligament from the extracted tooth; the quantity was small, and collagen fibers and blood vessels in the tissues may have affected the array results. The aim of the present study was to compare the differentially expressed genes between hDFCs and hPDLCs with differentiation capability. Cells obtained at P3 have a stronger differentiation capability. A total of 845 lncRNAs and 1,012 mRNAs were detected to be differentially expressed in hDFCs compared with hPDLCs. By comparing the expression of lncRNAs and mRNAs in hDFCs and hPDLCs, the present results identified variation in the expression levels of lncRNAs and mRNAs during the development of hDFCs. The results also indicated that upregulated lncRNAs and mRNAs were predominant during the development of hDFCs, and differentially expressed mRNAs and lncRNAs were used as candidates to screen the key genes associated with the differentiation of hDFCs by bioinformatics analysis. The RT-qPCR results confirmed the reliability of the microarray data. Markedly differential expression levels between the two types of cells indicated that lncRNAs may serve crucial roles in the differentiation and development of hDFCs.

lncRNAs may be categorized as sense, antisense, intronic, intergenic and bidirectional (25). Among them, ASlncRNAs are a type of endogenous lncRNA that complement other transcripts. Antisense transcription is a common phenomenon 
Table III. Top 10 downregulated and upregulated lncRNAs of human dental follicle cells compared with human periodontal ligament cells.

A, Downregulated

\begin{tabular}{lc}
\hline lncRNA & Log2 fold change \\
\hline G005378 & -55.6292005 \\
BC017988 & -20.5510994 \\
FGF13-AS1 & -18.1455729 \\
RP11-368I23.4 & -17.8927488 \\
LINC00473 & -16.2682486 \\
RP11-313F23.4 & -14.3828901 \\
RN7SKP240 & -13.2776605 \\
AP000619.5 & -11.8151652 \\
G075702 & -11.6414154 \\
RP11-256I23.3 & -10.831092 \\
\hline
\end{tabular}

B, Upregulated

\begin{tabular}{lc}
\hline lncRNA & Log2 fold change \\
\hline LINC00944 & 43.9000497 \\
AK055386 & 33.9312365 \\
G048345 & 33.5797092 \\
LOC101929504 & 33.5392967 \\
LINC01021 & 21.9723651 \\
APCDD1L-AS1 & 20.0893837 \\
RP11-13N12.1 & 17.314853 \\
LOC100506457 & 17.1318902 \\
uc.176 & 14.6428966 \\
G060456 & 13.6926091
\end{tabular}

lncRNA, long non-coding RNA; FGF13-AS1, fibroblast growth factor 13-antisense RNA 1; APCDD1L-AS1, APC down-regulated 1 like-antisense RNA 1.

in humans, and is based on whether the antisense RNA acts in cis or in trans. ASIncRNAs regulate gene expression at the transcriptional or post-transcriptional level via a number of biological mechanisms, including RNA-DNA interaction (chromatin remodeling), transcription interference and RNA-RNA interaction in the nucleus/cytoplasm. ASlncRNAs can serve as scaffolds between DNA and proteins, and participate in disease processes by assisting with interactions between ASIncRNAs, DNA and proteins (26). The current microarray results indicated the ASlncRNAs and nearby mRNAs that may regulate important biological processes. For example, the lncRNA NR_033932 was identified as one of the transcripts of IncRNA repulsive guidance molecule $b$ (RGMB)-AS1, which was revealed to be upregulated in hDFCs compared with hPDLCs. IncRNA RGMB-AS1 is located in the antisense region of RGMB, and RGMB carries the exon of the IncRNA RGMB-AS1 gene in the reverse strand and orientation of its intron region. IncRNA RGMB-AS1 silencing suppresses lung adenocarcinoma and hepatocellular
Table IV. Top 10 downregulated and upregulated mRNAs of hDFCs compared with hPDLCs.

\begin{tabular}{lc}
\hline A, Downregulated & \\
\hline mRNA & Log2 fold change \\
\hline KRT5 & -105.4128548 \\
SOX11 & -85.9063061 \\
LRP1B & -57.6366695 \\
SLC7A3 & -46.7530193 \\
CTAG2 & -42.1623478 \\
SLITRK6 & -41.7426552 \\
SPATA22 & -36.689978 \\
SHISA2 & -35.1885461 \\
LAMP3 & -31.6925565 \\
MMP13 & -25.9685676 \\
\hline
\end{tabular}

B, Upregulated

\begin{tabular}{lc}
\hline mRNA & Log2 fold change \\
\hline RPTN & 69.3550374 \\
PSG2 & 57.7064349 \\
ADRA2A & 56.7419608 \\
SOST & 55.5946956 \\
FLG & 55.4287499 \\
KCNB1 & 34.5671271 \\
SLC14A1 & 31.308326 \\
MFAP5 & 29.633329 \\
PSG5 & 27.7407034 \\
PSG7 & 25.9549841 \\
\hline
\end{tabular}

KRT5, keratin 5; SOX11, SRY-box 11; LRP1B, LDL receptor related protein 1B; SLC7A3, solute carrier family 7 member 3; CTAG2, cancer/testis antigen 2; SLITRK6, SLIT and NTRK like family member 6; SPATA22, spermatogenesis associated 22; SHISA2, shisa family member 2; LAMP3, lysosomal associated membrane protein 3; MMP13, matrix metallopeptidase 13; RPTN, repetin; PSG2, pregnancy specific $\beta$-1-glycoprotein 2; ADRA2A, adrenoceptor $\alpha$ 2A; SOST, sclerostin; FLG, filaggrin; KCNB1, potassium voltage-gated channel subfamily B member 1 ; SLC14A1, solute carrier family 14 member 1 ; MFAP5, microfibril associated protein 5; PSG5, pregnancy specific $\beta$-1-glycoprotein 5; PSG7, pregnancy specific $\beta$-1-glycoprotein 7.

carcinoma cell proliferation, migration and invasion, and leads to cell cycle arrest at the $\mathrm{G}_{1} / \mathrm{G}_{0}$ phase $(27,28)$. IncRNA T152410 was identified to be upregulated in hDFCs compared with hPDLCs. The nearby gene SMAD specific E3 ubiquitin protein ligase 2 (SMURF2) is a member of the HECT family of E3 ubiquitin ligases that regulate the polarity of cells during embryonic development and other signaling pathways for osteoblast migration, proliferation and differentiation. Notably, the anti-proliferative effect of SMURF2 siRNA is mediated by arresting cells in the $\mathrm{G}_{0} / \mathrm{G}_{1}$ phase, similar to the lncRNA RGMB-AS1 (29,30). This suggests that these two ASIncRNAs may promote hDFC migration, proliferation and differentiation by regulating the cell cycle. 

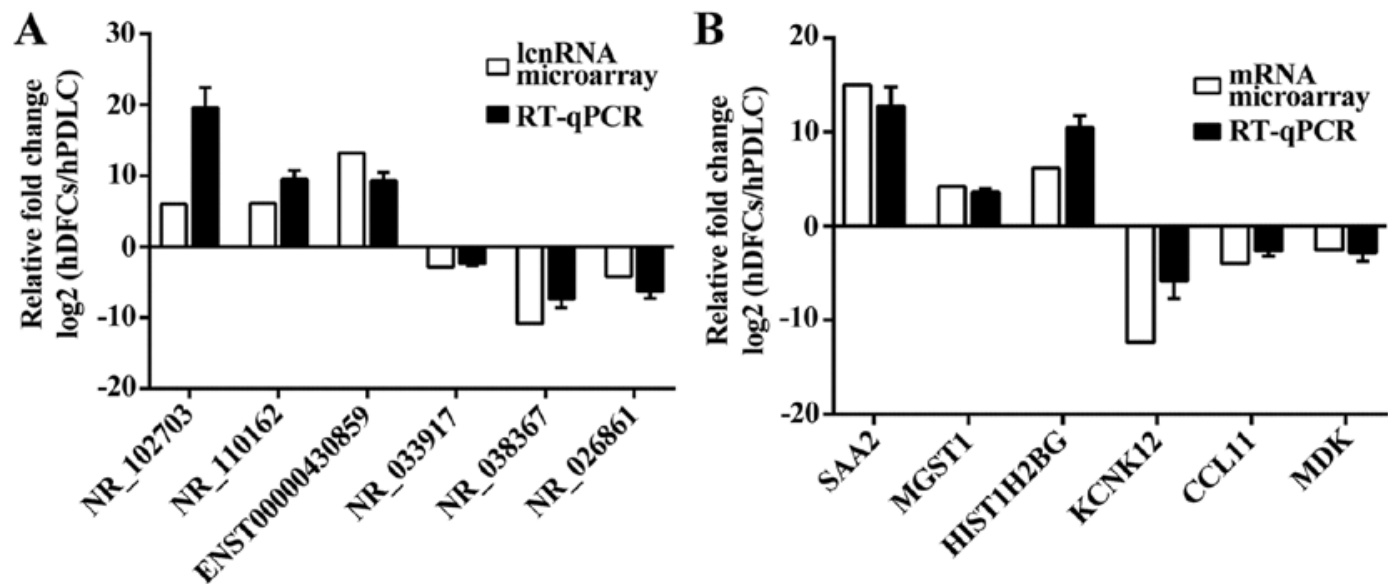

Figure 3. Validation of the microarray results using RT-qPCR. (A) Comparative expression levels of lncRNAs between hDFCs and hPDLCs obtained by microarray and RT-qPCR. (B) Comparative expression levels of mRNA between hDFCs and hPDLCs obtained by microarray and RT-qPCR. The microarray analyses were normalized and the RT-qPCR results were calculated using the $2^{-\triangle \Delta C q}$ method. SAA2, serum amyloid A2; MGST1, microsomal glutathione S-transferase 1; HIST1H2BG, histone cluster 1 H2B family member g; KCNK12, potassium two pore domain channel subfamily K member 12; CCL11, C-C motif chemokine ligand 11; MDK, midkine; RT-qPCR, reverse transcription-quantitative polymerase chain reaction; lncRNA, long non-coding RNA; hDFC, human dental follicle cell; hPDLC, human periodontal ligament cell.

$\mathbf{A}_{\substack{(179) \text { single-organism } \\ \text { developmental process }}}$ developmental process
(181) developmental process

(198) response to stimulus (205) single-multicellular organism process

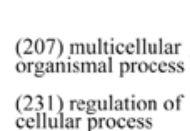

GO biological process classification

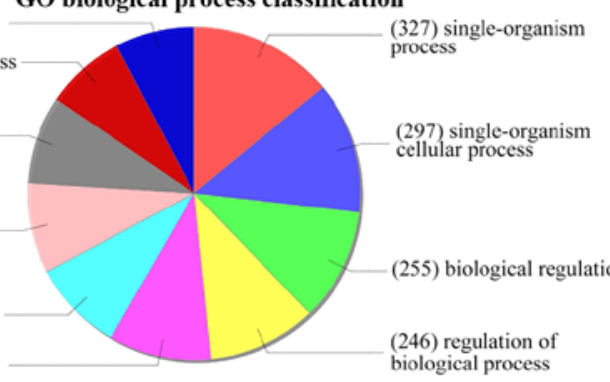

C

(15) actin cytoskeleton

GO cellular component classification

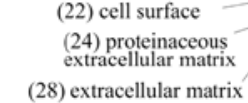

ular matrix

(45) integral component of plasma membrane

(46) extracellular space (47) intrinsic componen of plasma membrane

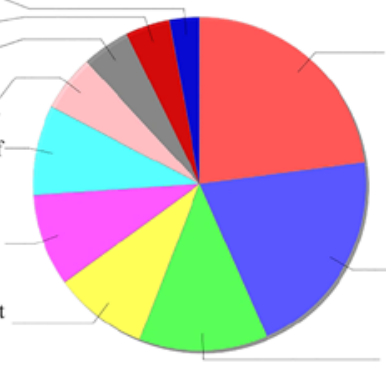

(118) extracellular region

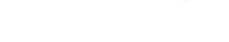

$\mathbf{E}$

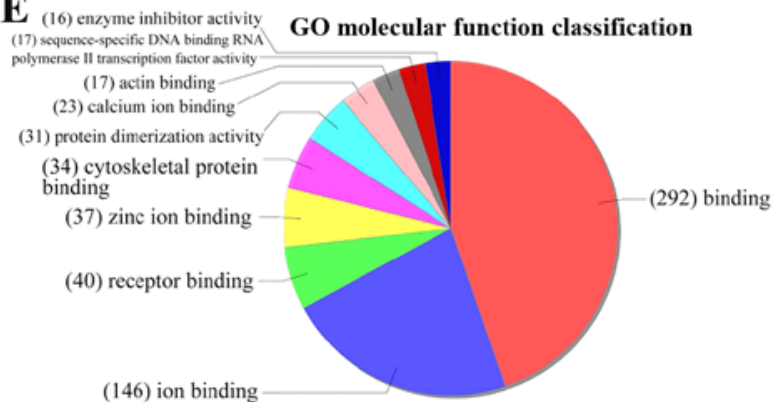

B

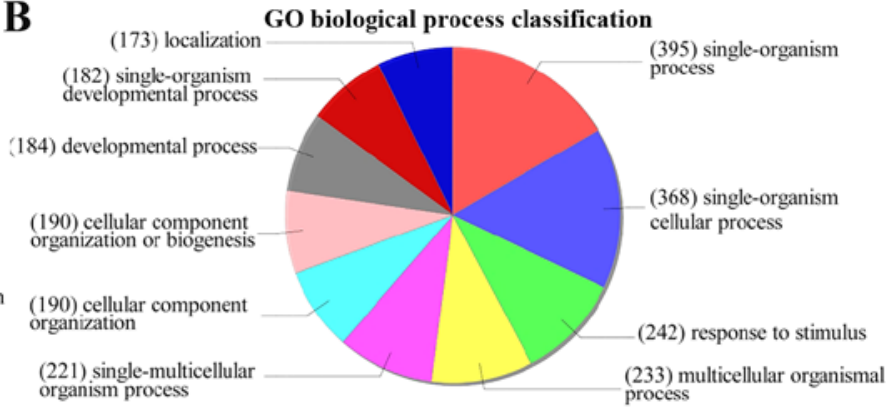

D

GO cellular component classification

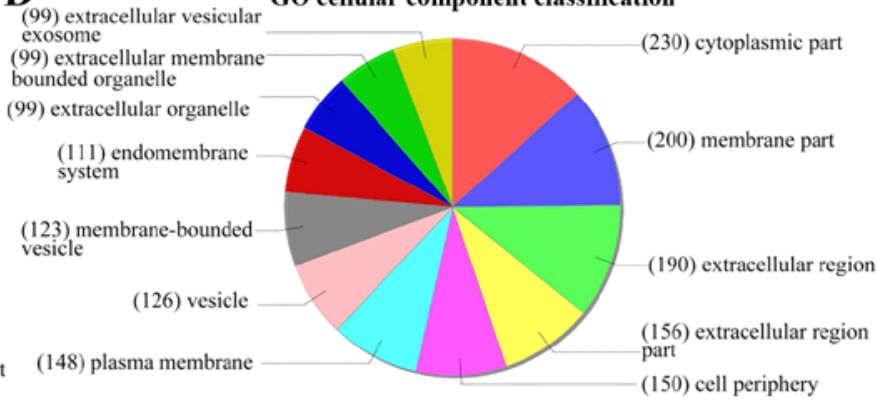

F

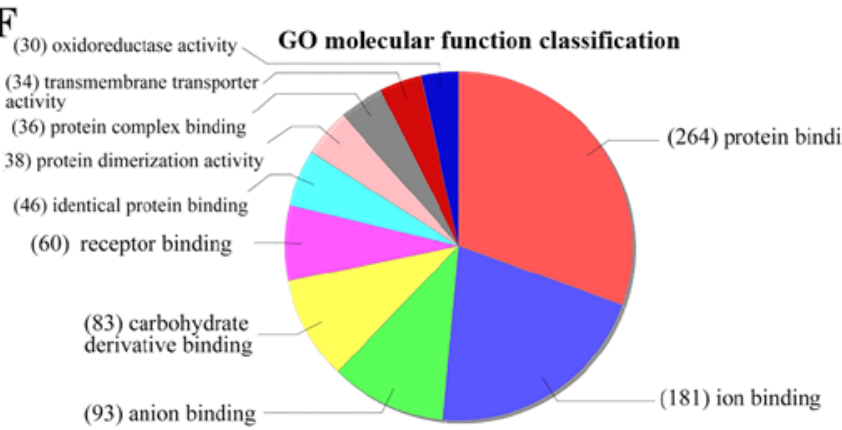

Figure 4. GO analysis of human dental follicle cells compared with human periodontal ligament cells. GO biological process classification of (A) downregulated and (B) upregulated mRNAs. GO cellular component classification of (C) downregulated and (D) upregulated mRNAs. GO molecular function classification of (E) downregulated and (F) upregulated mRNAs. GO, gene ontology.

lncRNAs may exert effects by controlling the transcriptional regulation of nearby coding genes (31). Subgroup analysis of the microarray demonstrated that a number of differentially expressed lincRNAs located near mRNAs 

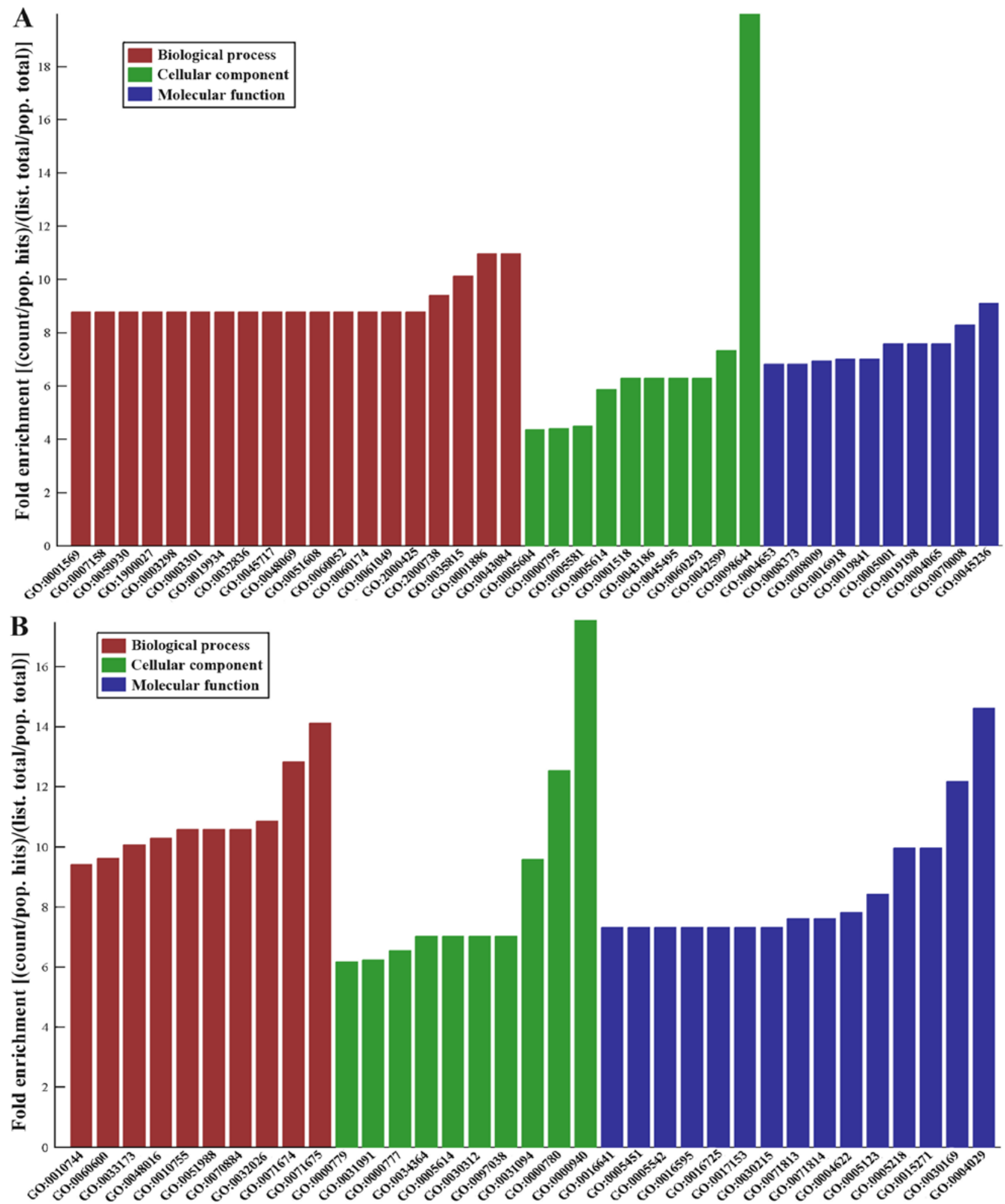

Figure 5. Significant GO terms of differentially expressed mRNAs of hDFCs compared with hPDLCs. (A) Significant GO terms of downregulated mRNAs identified in hDFCs compared with hPDLCs. (B) Significant GO terms of upregulated mRNAs identified in hDFCs compared with hPDLCs. GO, gene ontology; hDFCs, human dental follicle cells; hPDLCs, human periodontal ligament cells.

are associated with tissue development. For example, lncRNA ENST00000512129 and nearby mRNA lymphoid enhancer binding factor 1 (LEF1) were downregulated in hDFCs compared with hPDLCs. As a key mediator of the Wnt/ $\beta$-catenin signaling pathway and epithelial-mesenchymal interaction, LEF1 has been revealed to regulate incisor development. LEF1 also serves a key role in stem cell maintenance along with SOX2 and paired like homeodomain 2 (32,33). lncRNA ENST00000540293 and some nearby matrix metalloproteinase (MMP) mRNAs were revealed to be downregulated in hDFCs. MMPs are effective proteolytic mediators during ECM remodeling, and regulate the Notch signaling pathway, which is involved in differentiation. MMPs are able to regulate the necessary changes 
A

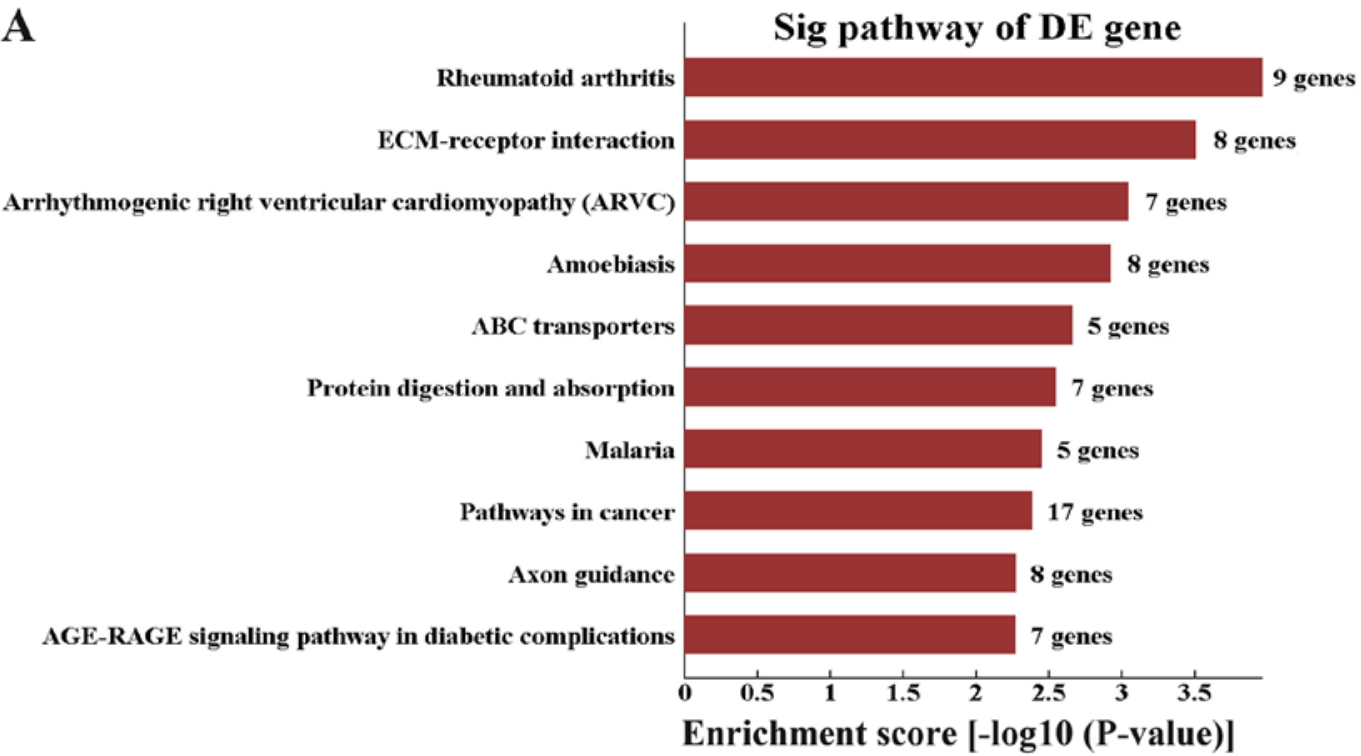

B

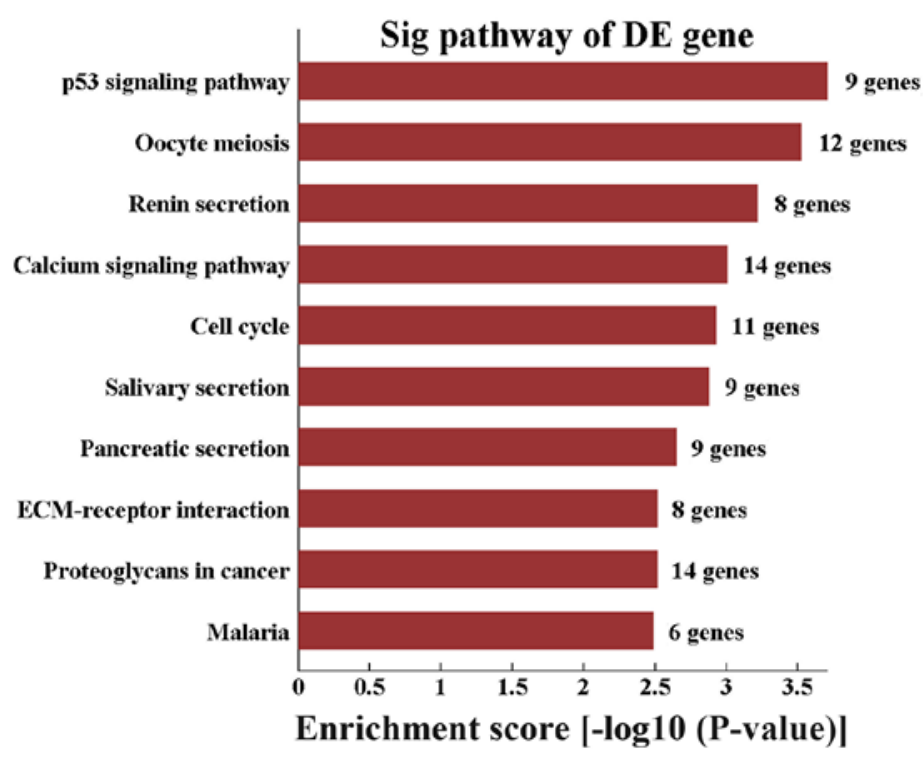

Figure 6. Top 10 pathways of DE mRNAs ranked by enrichment score. (A) Top ten pathways of downregulated mRNAs ranked by enrichment score. (B) Top ten pathways of upregulated mRNAs ranked by enrichment score. Sig, significant; DE, differentially expressed.

in the microenvironment, and overexpression of MMPs increases cell differentiation during development by altering the bioavailability of chemokines and cytokines that affect stem cell function (34). These results could be explained by evidence that lncRNAs regulate the expression of neighboring genes to promote cellular survival and differentiation (35).

Pathway analysis revealed that the top three downregulated pathways were 'rheumatoid arthritis', 'ECM-receptor interaction' and 'arrhythmogenic right ventricular cardiomyopathy (ARVC)'. In addition, the top three upregulated pathways were 'p53 signaling pathway', 'oocyte meiosis' and 'renin secretion'. Among these pathways, the p53 pathway is a vital signaling pathway in tumor biology. The expression level of p53 is high during early embryonic development. p53 serves an important role in self-renewal and human embryonic stem cell differentiation by regulating specific target genes or miRNAs and reactivating developmental pathways for tissue regeneration (36). The calcium pathway is a ubiquitous intracellular signaling pathway that participates in numerous cellular processes, including cell proliferation, differentiation and apoptosis. As an intracellular messenger, calcium serves an important role in the cellular signaling pathways at different stages of stem cell differentiation (37). Upregulation of calcium-mediated signaling is essential for the maintenance of stem cells. The current results indicated that these pathways may be involved in the development of hDFCs.

In addition, $\mathrm{CNC}$ analysis revealed a potential regulatory network between IncRNAs and mRNAs, and the mRNAs selected for CNC analysis were closely associated with the pathways in the development process of stem cells $(38,39)$. Among the lncRNA-mRNA pairs, IncRNA uc021sxs.1 was differentially expressed in hDFCs compared with hPDLCs and was correlated with Dickkopf-1 (DKK1). DKK1 serves as an antagonist of the canonical Wnt signaling pathway by binding to the Wnt receptor Lrp5/6, which is central to embryonic and adult bone development (40). The IncRNA ENST00000609146 was upregulated in hDFCs compared with hPDLCs and it was negatively correlated with Wnt5a. Wnt5a regulates a variety of biological processes, 

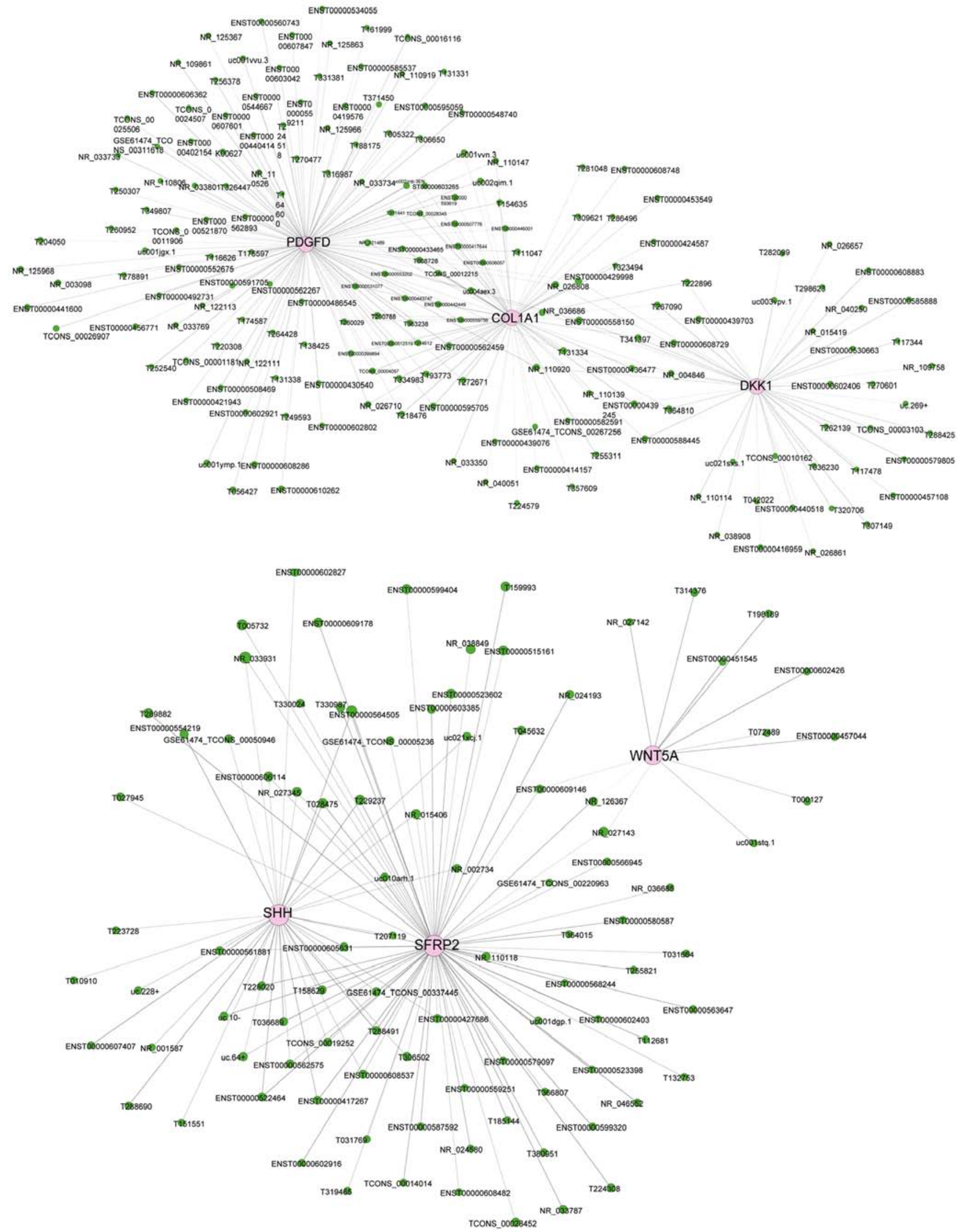

Figure 7. Part of the coding-non-coding gene co-expression network. Pink nodes represent mRNAs and green nodes represent long non-coding RNAs. Solid lines indicate a positive correlation and dashed lines represent a negative correlation.

including proliferation, differentiation, migration, adhesion and polarity (41). Wnt5a appears to serve important roles in the fate of DFSCs in the development and regeneration of the periodontium. Wnt5a can directly or indirectly activate the canonical Wnt 

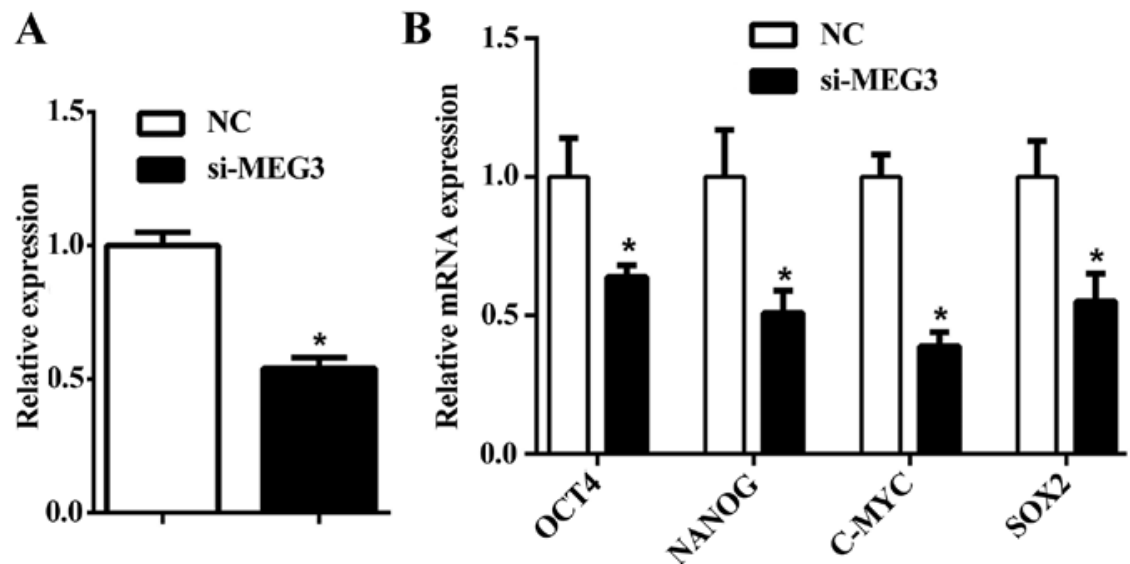

Figure 8. Expression of pluripotency-associated genes detected by RT-qPCR. (A) The transfection efficiency of MEG3-siRNA. (B) Decreased expression levels of pluripotency-associated genes were revealed following knockdown of MEG3 by siRNA for $72 \mathrm{~h}$. Data are presented as the mean \pm standard deviation (n=3). "P<0.05 vs. respective NC. MEG3, maternally expressed 3; siRNA, small interfering; NC, negative control; OCT4, POU class 5 homeobox 1; NANOG, Nanog homeobox; SOX2, SRY-box 2.

signaling pathway to promote mesendoderm differentiation (42). The correlations between these IncRNAs and mRNAs indicate that lncRNA uc021sxs.1 and lncRNA ENST00000609146 may be involved in associated signaling pathways by regulating mRNA expression. In addition, the results suggest that the activation of the Wnt signaling pathway may serve a critical role in hDFC development.

Although numerous studies have been conducted to identify cell factors that maintain the pluripotency of periodontal cells (43), potential lncRNAs with similar functions remain unknown. The current results demonstrated that MEG3 may serve a role in the establishment or maintenance of the stem cell state.

A study conducted by Lee et al (44) reported that $1.49 \%$ of mRNAs in hDFCs were differentially expressed (fold change $>2.0$ or $<-2.0 ; \mathrm{P}<0.05)$ compared with hPDLCs. The current microarray results indicated that among a total of 16,531 mRNAs, the expression levels of 1,012 mRNAs $(6.12 \%)$ were significantly different in hDFCs compared with hPDLCs. Differences in the ages of participants and cell state may explain this discrepancy. Compared with the study by Lee et al (44), the current analysis predominantly focused on the potential lncRNAs instead of mRNAs, which may serve critical roles in hDFC differentiation.

The present results may promote further studies to investigate the functions of genes and the regulatory mechanisms in hDFCs and periodontal tissue, and may advance the use of stem cell-based therapies in regenerative medicine. Furthermore, more studies should be conducted to investigate the potential functions that differentially expressed lncRNAs serve in multiple biological processes of hDFCs and periodontal tissue.

\section{Acknowledgements}

Not applicable.

\section{Funding}

The present study was supported by the National Natural Science Foundation of China (grant no. 81170932) and the
Natural Science Foundation of Guangdong Province (grant no. 2015A030313083).

\section{Availability of data and materials}

The datasets used and/or analyzed during the current study are available from the corresponding author on reasonable request.

\section{Authors' contributions}

LW, LD and JL conceived and designed the experiments. LD performed the experiments. LW, LD and $\mathrm{HH}$ analyzed the data. LD drafted the manuscript. CP, XZ, and ZC performed the experiments, analyzed the data and revised the manuscript. All authors reviewed and approved the final manuscript.

\section{Ethics approval and consent to participate}

The protocol to acquire human tissues was approved by the Ethical Guidelines of the Ethics Committee of the Hospital of Stomatology, Sun Yat-Sen University (Guangzhou, China). Written informed consent was obtained from all patients and their parents.

\section{Patient consent for publication}

Not applicable.

\section{Competing interests}

The authors declare that they have no competing interests.

\section{References}

1. Cho MI and Garant PR: Development and general structure of the periodontium. Periodontol 2000 24: 9-27, 2000.

2. Itaya S, Oka K, Ogata K, Tamura S, Kira-Tatsuoka M, Fujiwara N, Otsu K, Tsuruga E, Ozaki M and Harada H: Hertwig's epithelial root sheath cells contribute to formation of periodontal ligament through epithelial-mesenchymal transition by TGF- $\beta$. Biomed Res 38: 61-69, 2017. 
3. Sowmya S, Chennazhi KP, Arzate H, Jayachandran P, Nair SV and Jayakumar R: Periodontal specific differentiation of dental follicle stem cells into osteoblast, fibroblast, and cementoblast. Tissue Eng Part C Methods 21: 1044-1058, 2015.

4. Seo BM, Miura M, Gronthos S, Bartold PM, Batouli S, Brahim J, Young M, Robey PG, Wang CY and Shi S: Investigation of multipotent postnatal stem cells from human periodontal ligament. Lancet 364: 149-155, 2004.

5. Menicanin D, Mrozik KM, Wada N, Marino V, Shi S, Bartold PM and Gronthos S: Periodontal-ligament-derived stem cells exhibit the capacity for long-term survival, self-renewal, and regeneration of multiple tissue types in vivo. Stem Cells Dev 23: 1001-1011, 2014

6. Guo S, Guo W, Ding Y, Gong J, Zou Q, Xie D, Chen Y, Wu Y and Tian W: Comparative study of human dental follicle cell sheets and periodontal ligament cell sheets for periodontal tissue regeneration. Cell Transplant 22: 1061-1073, 2013.

7. Kashi K, Henderson L, Bonetti A and Carninci P: Discovery and functional analysis of lncRNAs: Methodologies to investigate an uncharacterized transcriptome. Biochim Biophys Acta 1859: $3-15,2016$.

8. Ghosal S, Das S and Chakrabarti J: Long noncoding RNAs: New players in the molecular mechanism for maintenance and differentiation of pluripotent stem cells. Stem Cells Dev 22: 2240-2253, 2013.

9. Schmitz SU, Grote P and Herrmann BG: Mechanisms of long noncoding RNA function in development and disease. Cell Mol Life Sci 73: 2491-2509, 2016.

10. Aprea J and Calegari F: Long non-coding RNAs in corticogenesis: Deciphering the non-coding code of the brain. EMBO J 34 2865-2884, 2015.

11. Korostowski L, Sedlak N and Engel N: The Kcnqlot1 long non-coding RNA affects chromatin conformation and expression of Kcnq1, but does not regulate its imprinting in the developing heart. PLoS Genet 8: e1002956, 2012.

12. Lv J, Huang Z, Liu H, Liu H, Cui W, Li B, He H, Guo J, Liu Q, Zhang $\mathrm{Y}$ and $\mathrm{Wu} \mathrm{Q}$ : Identification and characterization of long intergenic non-coding RNAs related to mouse liver development. Mol Genet Genomics 289: 1225-1235, 2014.

13. Herriges MJ, Swarr DT, Morley MP, Rathi KS, Peng T, Stewart KM and Morrisey EE: Long noncoding RNAs are spatially correlated with transcription factors and regulate lung development. Genes Dev 28: 1363-1379, 2014.

14. Hassan MQ, Tye CE, Stein GS and Lian JB: Non-coding RNAs: Epigenetic regulators of bone development and homeostasis. Bone 81: 746-756, 2015

15. Chen L, Song Z, Huang S, Wang R, Qin W, Guo J and Lin Z: IncRNA DANCR suppresses odontoblast-like differentiation of human dental pulp cells by inhibiting wnt $/ \beta$-catenin pathway. Cell Tissue Res 364: 309-318, 2016

16. Jia Q, Chen X, Jiang W, Wang W, Guo B and Ni L: The regulatory effects of long noncoding RNA-ANCR on dental tissue-derived stem cells. Stem Cells Int 2016: 3146805, 2016.

17. Viale-Bouroncle S, Klingelhöffer C, Ettl T and Morsczeck C: The WNT inhibitor APCDD1 sustains the expression of $\beta$-catenin during the osteogenic differentiation of human dental follicle cells. Biochem Biophys Res Commun 457: 314-317, 2015.

18. Wei M, Zhang M, Adams A and Duan Y: JNK and AKT/GSK3 $\beta$ signaling pathways converge to regulate periodontal ligament cell survival involving XIAP. Biochem Biophys Res Commun 448 485-491, 2014

19. Livak KJ and Schmittgen TD: Analysis of relative gene expression data using real-time quantitative PCR and the 2(-Delta Delta C(T)) method. Methods 25: 402-408, 2001.

20. Ashburner M, Ball CA, Blake JA, Botstein D, Butler H, Cherry JM, Davis AP, Dolinski K, Dwight SS, Eppig JT, et al Gene ontology: Tool for the unification of biology. The gene ontology consortium. Nat Genet 25: 25-29, 2000.

21. Sellers ZP, Schneider G, Maj M and Ratajczak MZ: Analysis of the paternally-imprinted DLK1-MEG3 and IGF2-H19 tandem gene loci in NT2 embryonal carcinoma cells identifies DLK1 as a potential therapeutic target. Stem Cell Rev 14: 823-836, 2018.

22. Peng S, Cao L, He S, Zhong Y, Ma H, Zhang Y and Shuai C: An overview of long noncoding RNAs involved in bone regeneration from mesenchymal stem cells. Stem Cells Int 2018: 8273648, 2018.

23. Farzi-Molan A, Babashah S, Bakhshinejad B, Atashi A and Fakhr TM: Down-regulation of the non-coding RNA H19 and its derived miR-675 is concomitant with up-regulation of insulin-like growth factor receptor type 1 during neural-like differentiation of human bone marrow mesenchymal stem cells. Cell Biol Int 42: 940-948, 2018.
24. Gu X, Li M, Jin Y, Liu D and Wei F: Identification and integrated analysis of differentially expressed IncRNAs and circRNAs reveal the potential ceRNA networks during PDLSC osteogenic differentiation. BMC Genet 18: 100, 2017.

25. Kunej T, Obsteter J, Pogacar Z, Horvat S and Calin GA: The decalog of long non-coding RNA involvement in cancer diagnosis and monitoring. Crit Rev Clin Lab Sci 51: 344-357, 2014.

26. Tsai MC, Manor O, Wan Y, Mosammaparast N, Wang JK, Lan F, Shi Y, Segal E and Chang HY: Long noncoding RNA as modular scaffold of histone modification complexes. Science 329: 689-693, 2010.

27. Li P, Zhang G, Li J, Yang R, Chen S, Wu S, Zhang F, Bai Y, Zhao H, Wang Y, et al: Long noncoding RNA RGMB-AS1 indicates a poor prognosis and modulates cell proliferation, migration and invasion in lung adenocarcinoma. PLoS One 11: e150790, 2016.

28. Sheng N, Li Y, Qian R and Li Y: The clinical significance and biological function of lncRNA RGMB-AS1 in hepatocellular carcinoma. Biomed Pharmacother 98: 577-584, 2018.

29. Choi YH, Kim YJ, Jeong HM, Jin YH, Yeo CY and Lee KY: Akt enhances Runx2 protein stability by regulating Smurf2 function during osteoblast differentiation. FEBS J 281: 3656-3666, 2014.

30. David D, Jagadeeshan S, Hariharan R, Nair AS and Pillai RM: Smurf2 E3 ubiquitin ligase modulates proliferation and invasiveness of breast cancer cells in a CNKSR2 dependent manner. Cell Div 9: 2, 2014.

31. Li B, Chen P, Qu J, Shi L, Zhuang W, Fu J, Li J, Zhang X, Sun Y and Zhuang W: Activation of LTBP3 gene by a long noncoding RNA (lncRNA) MALAT1 transcript in mesenchymal stem cells from multiple myeloma. J Biol Chem 289: 29365-29375, 2014.

32. Sun Z, Yu W, Sanz Navarro M, Sweat M, Eliason S, Sharp T, Liu H, Seidel K, Zhang L, Moreno M, et al: Sox 2 and Lef-1 interact with Pitx2 to regulate incisor development and stem cell renewal. Development 143: 4115-4126, 2016.

33. Santiago L, Daniels G, Wang D, Deng FM and Lee P: Wnt signaling pathway protein LEF1 in cancer, as a biomarker for prognosis and a target for treatment. Am J Cancer Res 7: 1389-1406, 2017.

34. Kessenbrock K, Wang CY and Werb Z: Matrix metalloproteinases in stem cell regulation and cancer. Matrix Biol 44-46: 184-190, 2015.

35. Bunch $\mathrm{H}$ : Gene regulation of mammalian long non-coding RNA. Mol Genet Genomics 293: 1-15, 2018.

36. Levine AJ and Berger SL: The interplay between epigenetic changes and the $\mathrm{p} 53$ protein in stem cells. Genes Dev 31: 1195-1201, 2017 .

37. Tonelli FM, Santos AK, Gomes DA, da Silva SL, Gomes KN, Ladeira LO and Resende RR: Stem cells and calcium signaling. Adv Exp Med Biol 740: 891-916, 2012.

38. Liu Q, Hu CH, Zhou CH, Cui XX, Yang K, Deng C, Xia JJ, Wu Y, Liu LC and Jin Y: DKK1 rescues osteogenic differentiation of mesenchymal stem cells isolated from periodontal ligaments of patients with diabetes mellitus induced periodontitis. Sci Rep 5: 13142,2015

39. Xiang L, Chen M, He L, Cai B, Du Y, Zhang X, Zhou C, Wang C, Mao JJ and Ling J: Wnt5a regulates dental follicle stem/progenitor cells of the periodontium. Stem Cell Res Ther 5: 135, 2014.

40. Ou L, Fang L, Tang H, Qiao H, Zhang X and Wang Z: Dickkopf Wnt signaling pathway inhibitor 1 regulates the differentiation of mouse embryonic stem cells in vitro and in vivo. Mol Med Rep 13: 720-730, 2016.

41. Kikuchi A, Yamamoto H, Sato A and Matsumoto S: Wnt5a: Its signalling, functions and implication in diseases. Acta Physiol (Oxf) 204: 17-33, 2012.

42. Yu CY and Kuo HC: The trans-spliced long noncoding RNA tsRMST impedes human embryonic stem cell differentiation through WNT5A-mediated inhibition of the epithelial-to-mesenchymal transition. Stem Cells 34: 2052-2062, 2016

43. Liu X, Tan GR, Yu M, Cai X, Zhou Y, Ding H, Xie H, Qu F, Zhang R, Lam CU, et al: The effect of tumour necrosis factor-alpha on periodontal ligament stem cell differentiation and the related signaling pathways. Curr Stem Cell Res Ther 11: 593-602, 2016.

44. Lee HS, Lee J, Kim SO, Song JS, Lee JH, Lee SI, Jung HS and Choi BJ: Comparative gene-expression analysis of the dental follicle and periodontal ligament in humans. PLoS One 8: e84201, 2013.

This work is licensed under a Creative Commons Attribution-NonCommercial-NoDerivatives 4.0 International (CC BY-NC-ND 4.0) License. 